\title{
Insects found on Araujia species (Apocynaceae, Asclepiadoideae) in Argentina
}

\author{
Diego Leonardo CARPINTERO ${ }^{1,3}$ \& Daniel TESTONI ${ }^{2,3}$
}

\begin{abstract}
${ }^{1}$ División Entomología, Museo Argentino de Ciencias Naturales “Bernardino Rivadavia”. Av. Ángel Gallardo 470 (1405), Ciudad Autónoma de Buenos Aires, Argentina. dcarpint@macn.gov.ar ${ }^{2}$ Herbario BBB, Departamento de Biología, Bioquímica y Farmacia, Universidad Nacional del Sur. San Juan 670, Bahía Blanca, Argentina; daniel. testoni@uns.edu.ar ${ }^{3}$ Consejo Nacional de Investigaciones Científicas y Técnicas, Argentina CONICET.
\end{abstract}

\begin{abstract}
The results of a survey of the natural enemies of moth plant or "tasi", species of Araujia (Apocynaceae, Asclepiadoideae), present in Argentina are shown in this paper. 57 species of insects were recorded of which 17 are cited as natural enemies of these species for the first time. Their specificity was determined and their potential use as biological control agents for Araujia hortorum Fourn. was hypothesized. Pseudosphex noverca Schaus (Lepidoptera, Arctiidae), is recorded for the first time in the Buenos Aires province, Argentina.
\end{abstract}

Key Words: Araujia hortorum, Asclepiadoideae, natural enemies, biological control, Argentina.

Resumen: Insectos hallados en especies de Araujia (Apocynaceae, Asclepiadoideae) en Argentina. Los resultados de un estudio de los enemigos naturales de las "planta polilla" o "tasi", especies del género Araujia (Apocynaceae, Asclepiadoideae), que se encontraron en Argentina se presentan en este documento. Se registraron 57 especies de insectos, 17 de las cuales son citadas como enemigos naturales de estas plantas por primera vez. Se determinó su especificidad y se planteó la hipótesis de su uso potencial como agentes de control biológico para Araujia hortorum Fourn. La especie Pseudosphex noverca Schaus, (Lepidoptera, Arctiidae), se cita por primera vez para la provincia de Buenos Aires, Argentina.

Palabras clave: Araujia hortorum, Asclepiadoideae, enemigos naturales, control biológico, Argentina.

\section{INTRODUCTION}

Few systematic studies have previously been carried out on natural insect enemies of native plants in Argentina. The well known listings of Bosq (1937, 1940, 1943) and more recently of Cordo et al. (2004) put emphasis on species that are important in agricultural crops, as until now, that is the sector where most surveys have been conducted.

The present study is part of a project on the biological control of Araujia hortorum E.Fourn., an invasive species in New Zealand. A survey was undertaken during 2004-2008, in Argentina and neighbouring countries, of the insect species found on A. hortorum, as well as on related genera and species, that could be of potential use as biological control agents of this plant in New Zealand.

In preliminary reports Winks \& Fowler (2000) and Winks et al. (2004), a total of 19 insect species found on "moth plant" (A. hortorum) are cited in Argentina, data that was later included in the "Catálogo de Insectos Fitófagos de la Argentina" published by Cordo et al. (2004). Villamil et al. (2005) later added 35 species to the list of phytophagous insects and predators found on $A$. hortorum and other species of the same genus (A. angustifolia (Hook. \& Arn.) Decne., A. sericifera Brot., A. odorata (Hook. \& Arn.) Fontella \& Goyder and A. brachystephana (Griseb.) Fontella $\&$ Goyder). That list was the result of surveys undertaken in the south of Buenos Aires province and in the Argentine mesopotamia (provinces of Entre Ríos, Corrientes and Misiones). Only 10 of these species (found on five species of the moth plants studied) were identified ( $21 \%$ of the total number of species).

In the present research, the study area was extended to the north and centre of Argentina, but the focus was on the north of Buenos Aires province which is considered as the most important area in Argentina as most species of moth plant studied are found there, both in anthropogenic 


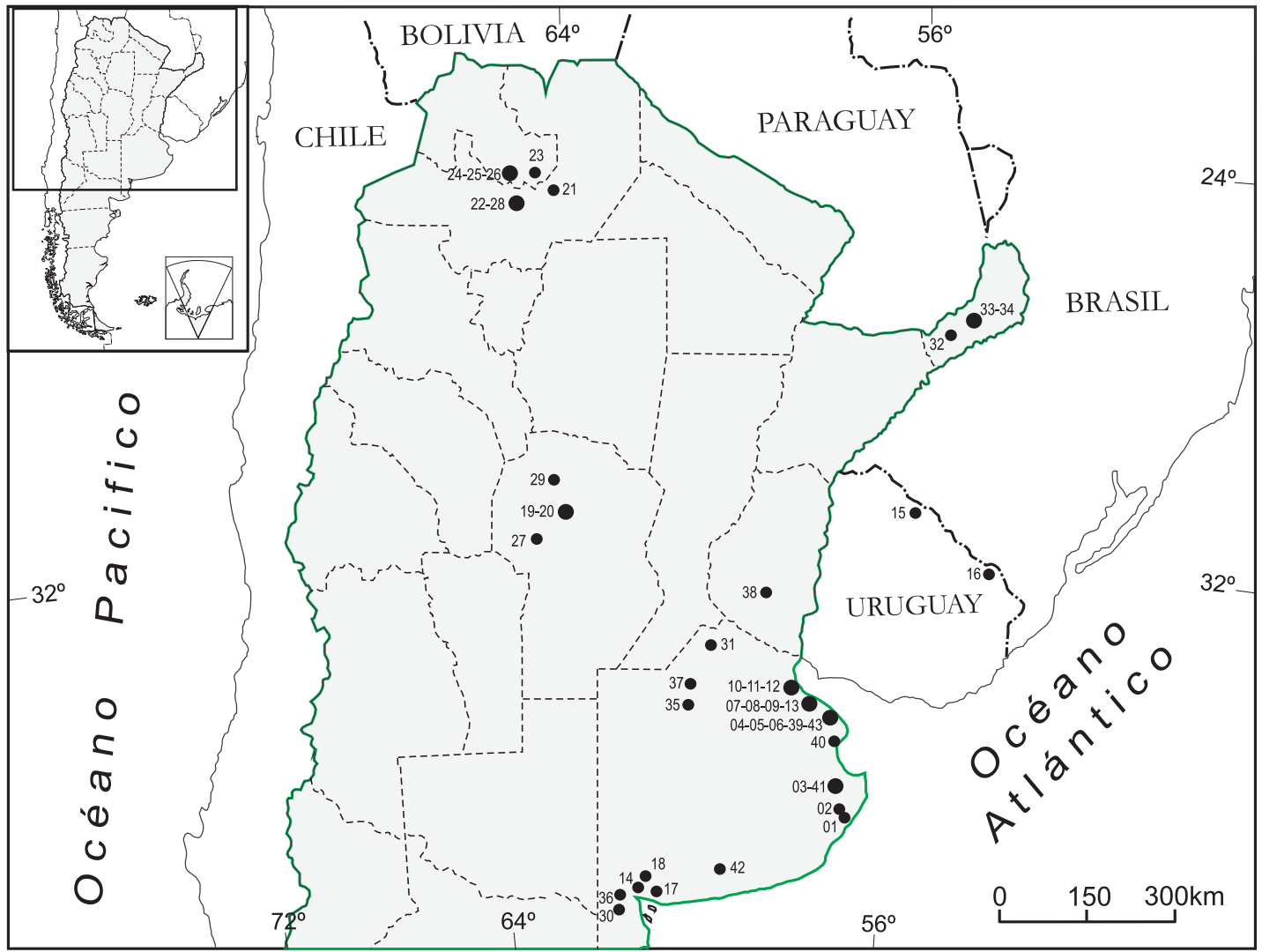

Fig. 1. Map of the sampling sites.

and pristine areas. Two other species of Araujia are included in this study: A. megapotamica (Spreng.) G.Don. and A. plumosa Schltdl. Some insect species are proposed as biological control agents of moth plants and their use is justified.

The authors have developed this research using the traditional approach, which considered Araujia and Morrenia as distinct genera (Meyer, 1944, here referred as sensu antico). Nevertheless, recently both genera have been brought together as Araujia (Rapini et al., 2011, here referred as sensu novo).

From the species found during the development of this project have been chosen by Landcare Research (the project sponsor) and preliminarily evaluated in laboratory, Colaspis argentinensis (Bechyné) (Coleoptera, Chrysomelidae) and Toxotrypana australis Blanchard (Diptera, Tephritidae).

\section{MATERIALS AND METHODS}

The specimens studied were collected in jars with $70 \%$ alcohol and were deposited in the
Entomology collection in the Museo Argentino de Ciencias Naturales "Bernardino Rivadavia" to be studied and stored.

The insects and plants, has been determined by comparison with reference collections, both in the collection of the Division of Entomology Museum of Natural History and the herbarium of the Universidad Nacional del Sur.

\section{Sampling sites (Fig. 1)}

\section{5-2006}

01. Pinamar, route $74 \mathrm{~km} \mathrm{5,} \mathrm{Buenos} \mathrm{Aires} \mathrm{province,}$ Argentina. On Araujia hortorum.

02. Pinamar, route $74 \mathrm{~km} \mathrm{14,} \mathrm{Buenos} \mathrm{Aires} \mathrm{province,}$ Argentina. On Araujia hortorum.

03. Ea La Luisa, route 56, $\mathrm{km} \mathrm{24,} \mathrm{Buenos} \mathrm{Aires} \mathrm{provin-}$ ce, Argentina. On Araujia hortorum.

04. Magdalena, $3 \mathrm{~km}$ south, Buenos Aires province Argentina. On Araujia hortorum.

05. Magdalena, route 11, $13 \mathrm{~km}$ north, Buenos Aires province, Argentina. On Araujia hortorum.

06. La Plata, Gonnet, Buenos Aires province Argentina. On Araujia hortorum.

07. Villa Elisa, Ecological Reserve, Buenos Aires province, Argentina. On Araujia hortorum. 
08. Berazategui, Gutiérrez, Buenos Aires, Argentina province. On Araujia odorata.

09. Punta Lara Reserve (Ensenada), Buenos Aires province, Argentina. On Araujia hortorum.

10. Quilmes, Laprida and La Plata Av., Buenos Aires province, Argentina. On Araujia odorata.

11. Quilmes, Calchaquí Av. and Irala street, Buenos Aires province, Argentina. On Araujia hortorum.

12. Quilmes, Amoedo and Gral. Mosconi Av., Buenos Aires province, Argentina. On Araujia angustifolia.

13. La Plata, Ringuelet, Buenos Aires province, Argentina. On Araujia hortorum.

14. Bahía Blanca, Buenos Aires province, Argentina. On Araujia hortorum.

15. Rivera, Uruguay. On Araujia brachystephana and A. odorata.

16. Rio Branco, Uruguay. On Araujia brachystephana and A. odorata.

\section{6-2008}

17. Bahía Blanca, Bajo Hondo, Buenos Aires province, Argentina. On Araujia odorata.

18. Bahía Blanca, Barrio Patagonia, Buenos Aires province, Argentina. On Araujia hortorum.

19. Córdoba, General Paz, prov. Córdoba, Argentina. On Araujia brachystephana.

20. Córdoba, General Paz, prov. Córdoba, Argentina. On Cynanchum sp.

21. General Güemes, prov. Salta, Argentina. Araujia odorata.

22. Cerro San Bernardo, prov. Salta, Argentina. On Philibertia latiflora.

23. Pampa Blanca, prov. Jujuy, Argentina. On Araujia odorata.

24. Dique Las Maderas, prov. Jujuy, Argentina. On Araujia odorata.

25. Dique Las Maderas, prov. Jujuy, Argentina. On Philibertia latiflora.

26. Dique Las Maderas, prov. Jujuy, Argentina. On Araujia plumosa.

27. Los Reartes, $5 \mathrm{~km}$ west, prov. Córdoba, Argentina. On Araujia odorata.

28. San Lorenzo, prov. Salta, Argentina. On Araujia odorata.

29. Deán Funes, prov. Córdoba, Argentina. On Araujia odorata.

30. Médanos, Lago Chapalcó, Buenos Aires province, Argentina. On Araujia odorata.

31. Pergamino ( $33^{\circ} 48^{\prime} 45.2$ ” S; 60²8' 35.8” W), Buenos Aires province, Argentina. On Araujia hortorum.

32. Candelaria ( $27^{\circ} 28^{\prime} 50.3^{\prime \prime} \mathrm{S}$; $55^{\circ} 44^{\prime} 49.0^{\prime \prime} \mathrm{W}$ ), prov. Misiones, Argentina. On Araujia sericifera.

33. Eldorado (26 23' 18.1” S; $53^{\circ} 53^{\prime} 39.6$ " W), prov. Misiones, Argentina. On Araujia sericifera.

34. Eldorado ( $26^{\circ} 23^{\prime} 38.4$ " S; $53^{\circ} 53^{\prime} 31.2$ ” W), prov. Misiones, Argentina. On Araujia sericifera.

35. Los Toldos ( $\left.34^{\circ} 51^{\prime} 38.4^{\prime \prime} \mathrm{S} ; 61^{\circ} 00^{\prime} 27.1^{\prime \prime} \mathrm{W}\right)$, Buenos Aires province, Argentina. On Araujia hortorum.

36. Médanos, Frigorífico Sur (38 $48^{\prime} 35.1^{\prime \prime}$ S; $62^{\circ} 40^{\prime}$ 59.2 " W), Buenos Aires province, Argentina. On Araujia odorata.
37. Junín ( $34^{\circ} 34^{\prime} 09.7 ”$ S; $60^{\circ} 57^{\prime} 36.5 ”$ W), Buenos Aires province, Argentina. On Araujia hortorum.

38. Gualeguay ( $33^{\circ} 09^{\prime} 24.0^{\prime \prime} \mathrm{S} ; 59^{\circ} 15^{\prime} 23.7^{\prime \prime} \mathrm{W}$ ), prov. Entre Ríos, Argentina. On Araujia brachystephana.

39. City Bell ( $\left.34^{\circ} 51^{\prime} 42.3^{\prime \prime} \mathrm{S} ; 58^{\circ} 03^{\prime} 47.5^{\prime \prime} \mathrm{W}\right)$, Buenos Aires province, Argentina. On Araujia hortorum.

40. Boca del Río Salado ( $35^{\circ} 45^{\prime} 04.5^{\prime}$ S; $57^{\circ} 22^{\prime} 54.7^{\prime \prime}$ W), Buenos Aires province, Argentina. On Araujia hortorum.

41. Ea. La Luisa, ruta $56 \mathrm{Km} 24\left(36^{\circ} 42^{\prime} 15.5^{\prime \prime} \mathrm{S} ; 57^{\circ}\right.$ 14' 26.5” W), Buenos Aires province, Argentina. On Araujia hortorum.

42. Tres Arroyos (38 22' 43.2” S; 60 15' 53.2” W), Buenos Aires province, Argentina. On Araujia hortorum.

43. Gonnet, Buenos Aires province, Argentina. On Araujia hortorum.

\section{RESULTS}

These are the final results of the survey of natural enemies of Araujia, after five years of field work (2004-2008). The authors have added 17 new natural enemies to the preliminary lists.

\section{Record of herbivorous insects reported on different species of Araujia in Argentina}

Featured here is the list of species found and published in the reports of the various campaigns conducted between 2000 and 2008

(1) Species cited in Winks \& Fowler (2000).

(2) Species cited in Villamil et al. (2005).

(3) Species cited in Villamil et al. (2006).

(4) Species cited here for the first time are shown in bold type.

NOTE: Unidentified species cited in Villamil et al. (2005) were shown with small letters (a, b, c).

\section{COLEOPTERA}

Anobiidae

a. sp. (2)

Comments. Probably the same species as (1). On Araujia hortorum.

1. Trycorynus subrutiliceps Pic (3)

Anthribidae

1. Araecerus fasciculatus (DeGeer) (4) (Fig. 9).

Cerambycidae

2. Acanthoderes jaspidea (Germain) (1)

3. Eupogonius petulans Melzer (1)

4. Eupromerella propinqua (Melzer) (3)

5. Hyperplatys argentinus Berg (1)

6. Neocorus ibidionoides (Serville) (3)

7. Urgleptes mancus (Melzer) (1) 
Chrysomelidae

8. Chlamisus hispidulus hispidulus (Klug) (3)

9. Colaspis argentinensis (Bechyné) (1) (3) (4)

2. Colaspis porosa Jacoby (4)

10. Cacoscelis melanoptera (Germain) (1)

Curculionidae

11. Araptus araujiae (Brèthes) (1) (3) (Scolytinae)

(4)

12. Araptus pubescens (Schedl) (3) (Scolytinae)

(4)

3. Asynonychus cervinus (Boheman) (4) b. curculioniform larvae? (2)

Comments. Probably the same species of

Scolytinae previously cited $(11,12)$. On Araujia hortorum and A. dorata.

4. Naupactus sp.1 (4)

5. Naupactus $\mathrm{sp.2}$ (4)

6. Naupactus sp.3 (4)

7. Naupactus sp.4 (4)

13. Rhyssomatus diversicollis Heller (1) (3)

14. Rhyssomatus pilosipes Heller (3) c. sp. (2)

Comments. Without any taxonomic specifica-

tions. On Araujia hortorum, A. angustifolia and

A. brachystephana.

Dasytidae

15. Astylus atromaculatus (Blanchard) (2) (4)

Meloidae

16. Tetraonyx propinquus Burmeister (3)

Tenebrionidae

8. Epitragus mucidus Berg (4)

\section{DIPTERA}

d. Pupae (did not emerge) (2)

Comments. Without any taxonomic specifications. On Araujia hortorum.

e. leaf miner? (2)

Comments. Without any taxonomic specifications. On Araujia hortorum.

vermiform larvae? (2)

Comments. Without any taxonomic specifications. On Araujia hortorum and A. angustifolia. Muscidae

sp. ? (2)

Comments. Without any taxonomic specifications. On Araujia hortorum.

Syrphidae

Pupae (2)

Comments. Without any taxonomic specifications. On Araujia odorata.

Tephritidae (Fig. 12)

9. Tomoplagia fiebrigi Hendel (4)

17. Toxotrypana australis Blanchard (3)

HEMIPTERA-HETEROPTERA

Coreidae:
18. Acanonicus hahni (Stal) (3) (4)

19. Anasa guttifera Berg (3)

20. Eubule glyphica Berg (2) (3)

21. Eubule sculpta (Perty) (1) (2) (3) (4) (Figs.

2,3)

10. Phthiacnemia picta (Drury) (Nymph) (4)

Lygaeidae

11. Lygaeus alboornatus Blanchard (4) (Fig. $6)$.

22. Oncopeltus unifasciatellus Hahn (2) (3) (4)

(Fig. 5).

23. Oncopeltus bergianus (Kirkaldy) (1) (3) (4)

(formerly stali) (Fig. 4).

Pentatomidae

12. Edessa sp. (Nymph) (4)

13. Nezara viridula L. (4)

Scutelleridae

14. Tetyra poecila Berg (4)

\section{HEMIPTERA-STERNORHYNCHA}

Aphididae

24. Aphis nerii Boyer de Fonscolombe (1) (2) (3)

(4) (Fig. 10).

25. Aphis gossypii Glover (dark green aphids) (2)

(3) (4) i.very small yellow aphids (2)

Comments. Probably a different species to Aphis nerii but further study is needed. On Araujia hortorum.

26. Myzus persicae (Sulzer) (3)

Lecaniidae

27. Saissetia oleae (Olivier) (3) (4)

Pseudoccidae

28. Pseudococcus sp. (mealy bugs) (2) (3)

\section{HEMIPTERA-COLEORHYNCHA}

Membracidae

15. Ceresa uruguayensis Berg (4) (Fig. 11).

\section{HYMENOPTERA}

Formicidae

29. Acromyrmex lundi Guérin (2)

30. Camponotus sp. (2) (3)

\section{LEPIDOPTERA}

Arctiidae (Fig. 16).

16. Pseudosphex noverca Schaus (4)

Comments. This is the first record of this species in Buenos Aires province.

Danaidae

31. Danaus erippus (Cramer) (3) (Figs. 7,8). j.monarch larvae (2)

Comments. Probably like the previous species.

On Araujia hortorum and A. angustifolia.

Hesperiidae

32. Pyrgus sp. (2) 

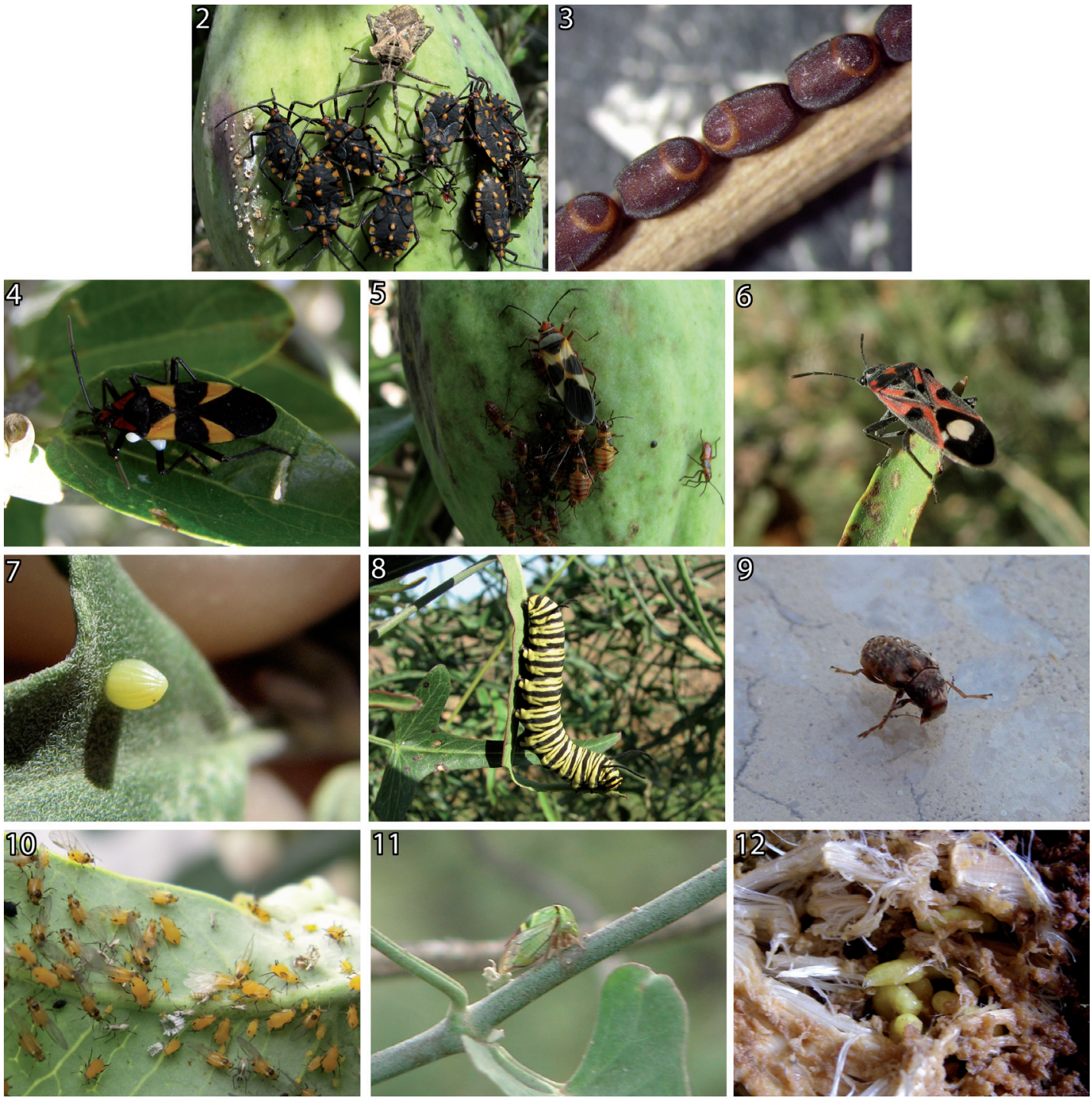

Figs. 2-12. 2. Adult and larvae of E. sculpta (Coreidae, Hemiptera) on fruit of A. hortorum. 3. Eggs of Eubule sculpta (Coreidae, Hemiptera) on stem of A. odorata. 4. Adult of Oncopeltus bergianus (Lygaeidae, Hemiptera) on leaf of A. hortorum. 5. Adult and nymphs of Oncopeltus unifasciatellus (Lygaeidae, Hemiptera) on fruit of A. hortorum. 6. Adult of Lygaeus alboornatus (Lygaeidae, Hemiptera) on leaf of A. brachystephana. 7. Egg of Danaus erippus (Danaidae, Lepidoptera) on leaf of $A$. odorata. 8. Nymph of $D$. erippus (Danaidae, Lepidoptera) on flower of A. odorata. 9. Araecerus fasciculatus (Anthribidae, Coleoptera) on A. sericifera. 10. Aphis nerii (Aphiidae, Hemiptera) on leaf of A. hortorum. 11. Ceresa uruguayensis (Membracidae, Hemiptera) on stem of $A$. odorata. 12. Larvae of Tephritidae (Diptera) on fruits of $A$. sericifera.

Noctuidae

33. Helicoverpa zea (Boddie) (2)

34. Rachiplusia nu (Genée) (2)

Sphyngidae

35. Erinnus ello (L.) (3)

36. Erinnus lassauxi (Boisduval) (3)

37. Erinnus obscura (F.) (3) k. Unidentified butterfly (2)

Comments. Without any taxonomic specifica- tions. On Araujia hortorum.

eruciform larvae (2)

Comments. Without any taxonomic specifications. On Araujia hortorum.

\section{ORTHOPTERA}

Proscopiidae

38. sp. (2) 


\section{PSOCOPTERA}

Psocidae

39. sp. (2)

Ectopsocidae

17. Ectopsocus (brisqsi group) prob. californicus (Banks) (4)

\section{THYSANOPTERA}

40. sp. (2) (3)

\section{Additional information}

List of parasites and predators recorded on Araujia species in Argentina.

\section{COLEOPTERA}

Carabidae

Lebia securigera Chaudoir (3)

Coccinellidae

Adalia bipunctata L. (4)

Azya luteipes Mulsant (4)

Coccinella ancoralis (Germain) (2) (3) (4)

Cycloneda sanguinea L. (4)

Harmonía axyridis (Pallas) (3)

Staphilinidae

Sp. (3)

\section{HEMIPTERA}

HETEROPTERA

Pentatomidae

Podisus aenescens (Stål) (4)

Reduviidae

Zelus leucogrammus (Perty) (4)

\section{HYMENOPTERA}

Mutillidae

Sp. (2)

Serie Parasítica (hosts: aphids, mealybugs, coleoptera larvae)

Sp. (2)

Pompilidae

Sp. (2)

\section{CONCLUSIONS}

\section{Results of the 2004-2008 survey}

Fifty seven species of phytophagous insects were recorded on the nine species studied of Araujia, and some other Asclepiadoideae, of which 46 were identified to species level and eight to the genus. Another 12 unidentified morphotypes cited in Villamil et al. (2005) were also included with brief comments.

Parasitic and predator species found on these plants during 2005-2008 were also surveyed and commented on briefly.
Of the total of 57 phytophagous species studied it is concluded that:

The following 24 species are not very important as they are polyphytophagous: Trycorynus subrutiliceps, Acanthoderes jaspidea, Eupogonius petulans, Hyperplatys argentinus, Urgleptes mancus, Chlamisus hispidulus hispidulus, Cacoscelis melanoptera, Astylus atromaculatus, Tetraonyx propinquus, Acanonicus hahni, Anasa guttifera, Acromyrmex sp., Pyrgus sp., Helicoverpa zea, Rachiplusia nu, Erinnus ello, Psocidae sp., Proscopidae sp., Araecerus fasciculatus, Epitragus mucidus, Phthia picta, Lygaeus alboornatus, Nezara viridula, Ectopsocus prob. californicus.

The following 17 species should be studied further to evaluate their potential as biological control agents: Tetyra poecila, Eupromerella propincua and Rhyssomatus pilosipes (for to be monospecific with Araujia odorata); Neocorus ibidionoides, Asynonychus cervinus and Erinnus obscura (for their specificity with Asclepiadoideae); Araptus pubescens, Toxotrypana australis and Erinnus lassauxi (for their specificity with Araujia odorata and $A$. brachystephana), Camponotus sp. (important as a symbiont with Pseudococcus sp.); Naupactus spp. 1, 2, 3, 4, Edessa sp., Tomoplagia fiebrigi and several species of THYSANOPTERA.

The final 16 species appear to be very important for future study on biological control: Oncopeltus bergianus, Rhyssomatus diversicollis, Colaspis argentinensis, Colaspis porosa, Araptus araujiae and Pseudosphex noverca (for their specificity with Araujia, sensu antico); Oncopeltus unifasciatellus, Eubule sculpta, Eubule glyphica and Danaus erippus (for being restricted to Araujia, sensu novo); Ceresa uruguayensis (for being restricted to Morrenia, sensu antico); Aphis nerii, Aphis gossypii, Myzus persicae, Saissetia oleae, Pseudococcus sp. (These are polyphytophagous but their populations and damage caused to the plants studied are very important, as well as their capacity as virus vectors).

\section{ACKNOWLEDGEMENTS}

This project was funded by Landcare Research, NZ. The participation of the authors was also supported by the Consejo Nacional de Investigaciones Científicas y Técnicas (CONICET). Special thanks to Dra. Soledad Villamil and Dr. Carlos Villamil for their great contribution to the scientific enrichment of this study and for critically reading the manuscript. 
Table 1. Presence of insects recorded on different species of Araujia. X. Pest/host associations mentioned in Winks \& Fowler (2000). Y. Pest/host associations mentioned in Villamil et al. (2005). Z. Pest/host associations mentioned in Villamil et al. (2006). Z. Pest/host associations mentioned here for the first time. 1. Pests on more than one species of Araujia or Morrenia (both in sensu antico). 2. Pests found only on different species but only on Asclepiadaceae. 3. Specific pests on one of the moth plants studied (monospecific pests). ?. No data. Species without numbers are polyphytophagous (also present in other plants not Asclepiadaceae).

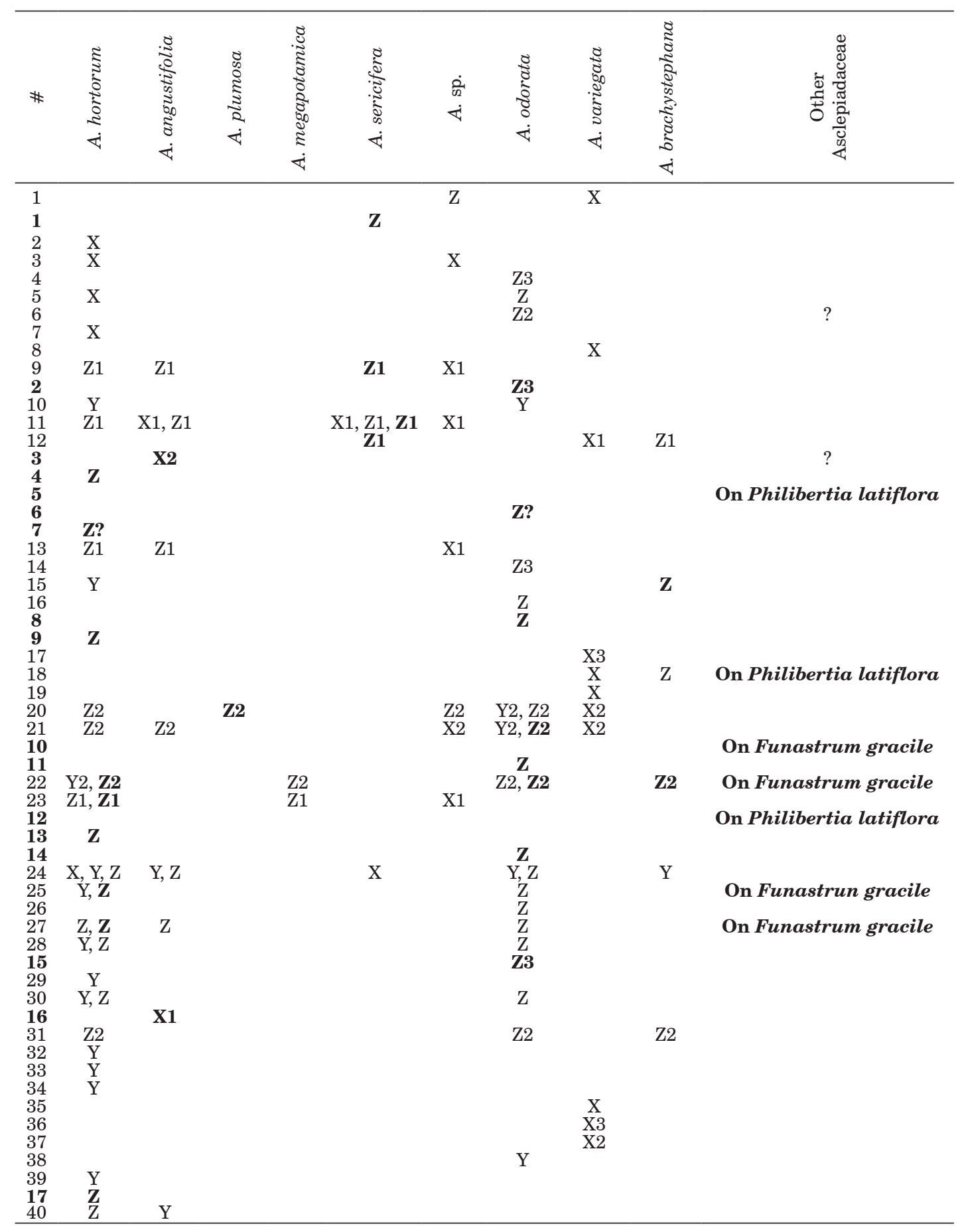


Table 2. Characteristics and potentialities of herbivorous insect populations found on different species of Araujia. Definitions of frequency categories according to Winks et al. (2004): rare: fewer than five individuals collected in total. occasional: a total of 5-24 individuals collected, or present at fewer than five sites. common: 25 or more specimens collected and present at five or more sites. abundant: more than 200 individuals collected and present at 10 or more sites. These data may be relative because damage caused by, for example, 10 larvae of Rhyssomatus, is greater than damage caused by 500 Aphis nerii. Different feeding habits according to Winks et al. (2004): Foliage feeders. Flower/Fruit feeders. Sap feeders. Stem borers. Bosq (1937), Bosq (1940), Bosq (1943), Cordo et al. (2004): Refers to added information found in the bibliography. rare?: supposition based on previous knowledge of the group. ??: unknown. Comments: Potentiality as a Biological Control Agent for Moth Plant.

\begin{tabular}{|c|c|c|c|c|}
\hline $\begin{array}{l}\text { Species } \\
\text { number }\end{array}$ & Collecting site & Frequency & Feeding habits & Comments \\
\hline 1 & - & common? & Stem borers (Bosq, 1943) & Polyphytophagous. \\
\hline 1 & 31 & occasional & Bark's fruits feeders & Polyphytophagous. \\
\hline 2 & - & rare? & Stem borers? & Polyphytophagous. \\
\hline 3 & - & rare? & Stem borers (Bosq, 1943) & Polyphytophagous. \\
\hline 4 & - & rare? & Stem borers? & $\begin{array}{l}\text { Need to be studied. May be important due to } \\
\text { its specificity (on A. odorata). }\end{array}$ \\
\hline 5 & - & rare? & Stem borers? & Polyphytophagous. \\
\hline 6 & - & rare? & Stem borers? & $\begin{array}{l}\text { Need to be studied. May be important. } \\
\text { Restricted to some Asclepiadoideae. }\end{array}$ \\
\hline 7 & - & rare? & Stem borers (Bosq, 1943) & Polyphytophagous. \\
\hline 8 & - & $? ?$ & Foliage feeders? & Polyphytophagous. \\
\hline 9 & $04,06,07,32$ & occasional & Foliage feeders & $\begin{array}{l}\text { Important. Restricted to Araujia (sensu } \\
\text { antico) }\end{array}$ \\
\hline 2 & 17 & occasional & Foliage feeders & Need to be studied. May be important. \\
\hline 10 & - & $? ?$ & Foliage feeders? & Polyphytophagous. \\
\hline 11 & $03,05,34$ & common & Fruit feeders & $\begin{array}{c}\text { Very important. Restricted to Araujia (sensu } \\
\text { antico). }\end{array}$ \\
\hline 12 & 34 & common & Fruit feeders (Bosq, 1943) & $\begin{array}{l}\text { Could be important. Restricted to Morrenia } \\
\text { (sensu antico). }\end{array}$ \\
\hline 3 & 06 & common & Foliage feeders & Polyphytophagous. \\
\hline 4 & 06 & rare & Foliage feeders & Only one specimen. May be occasional. \\
\hline 5 & 22 & rare & Foliage feeders & Only one specimen. May be occasional. \\
\hline 6 & 23 & rare & Foliage feeders & Only one specimen. May be occasional. \\
\hline 7 & 35 & rare & Foliage feeders & Only one specimen. May be occasional. \\
\hline 13 & $04,06,07$ & occasional & Fruit feeders & $\begin{array}{l}\text { Very important. Restricted to Araujia (sensu } \\
\text { antico). }\end{array}$ \\
\hline 14 & - & occasional? & Fruit feeders (Bosq, 1943) & $\begin{array}{c}\text { Could be important. Restricted to Araujia } \\
\text { odorata. }\end{array}$ \\
\hline 15 & 19,28 & common & $\begin{array}{l}\text { Flower / fruit feeders (Bosq, } \\
\text { 1943) }\end{array}$ & Polyphytophagous. \\
\hline 16 & - & $? ?$ & Flower feeders? & Polyphytophagous. \\
\hline 8 & 23 & rare & Flower feeders? & Only one specimen. May be occasional. \\
\hline 9 & 41 & rare & Fruit feeder & Could be important. \\
\hline 17 & - & $? ?$ & Fruit feeders? & $\begin{array}{l}\text { Need to be studied. May be important due to } \\
\text { its specificity. }\end{array}$ \\
\hline 18 & 22 & rare & Sap feeders & Polyphytophagous. \\
\hline 19 & - & common? & Sap feeders & Polyphytophagous. \\
\hline 20 & 1426 & common & Sap feeders & Very important. Restricted to Araujia species. \\
\hline 21 & $\begin{array}{l}07,21,23,28,29 \\
\quad 17,30,36,27\end{array}$ & common & Sap feeders & Very important. Restricted to Araujia species. \\
\hline 10 & 20 & rare & Sap feeders & Polyphytophagous. \\
\hline 11 & 21,28 & rare & Sap feeders & Polyphytophagous. \\
\hline 22 & $\begin{array}{l}15,16,20,24,17 \\
18,30,37,38\end{array}$ & common & Sap feeders & Very important. Restricted to Araujia species. \\
\hline 23 & $\begin{array}{l}01,02,07,16,39 \\
40,41,42\end{array}$ & common & Sap feeders & $\begin{array}{l}\text { Very important. Restricted to Araujia (sensu } \\
\text { antico). }\end{array}$ \\
\hline 12 & 22 & rare & Sap feeders & A single specimen. Polyphytophagous. \\
\hline 13 & 31 & rare & Sap feeders & A single specimen. Polyphytophagous. \\
\hline 14 & 27 & rare & Sap feeders & A single specimen. Polyphytophagous. \\
\hline 24 & $06,07,11,12,43$ & abundant & Sap feeders & $\begin{array}{c}\text { Polyphytophagous but very important as a } \\
\text { virus vector. }\end{array}$ \\
\hline 25 & 11,43 & common & Sap feeders & $\begin{array}{l}\text { IDEM A. nerii but with less important popula- } \\
\text { tions. }\end{array}$ \\
\hline 26 & - & common? & Sap feeders & $\begin{array}{l}\text { IDEM A. nerii but with less important popula- } \\
\text { tions. }\end{array}$ \\
\hline 27 & 11,43 & common & Sap feeders & $\begin{array}{l}\text { IDEM A. nerii but with less important popula- } \\
\text { tions. }\end{array}$ \\
\hline 28 & $08,10,11$ & abundant & Sap feeders & IDEM A. nerii. With important populations. \\
\hline
\end{tabular}


Table 2. Cont.

\begin{tabular}{|c|c|c|c|c|}
\hline $\begin{array}{l}\text { Species } \\
\text { number }\end{array}$ & Collecting site & Frequency & Feeding habits & Comments \\
\hline 15 & 27 & occasional & Sap feeders & Need to be studied. May be important. \\
\hline 29 & - & abundant? & Foliage feeders & Polyphytophagous. \\
\hline 30 & 06 & abundant & See comments & $\begin{array}{l}\text { Many species of this genus, and also other } \\
\text { ants, are symbionts of mealy bugs. }\end{array}$ \\
\hline 16 & 06 & common & Foliage feeders & $\begin{array}{l}\text { Need to be studied. May be important due to } \\
\text { its specificity. }\end{array}$ \\
\hline 31 & 09 & occasional & Foliage feeders & Very important. Restricted to Araujia species. \\
\hline 32 & - & ?? & ?? & Polyphytophagous. \\
\hline 33 & - & $? ?$ & ?? & Polyphytophagous. \\
\hline 34 & - & $? ?$ & $? ?$ & Polyphytophagous. \\
\hline 35 & - & ?? & Foliage feeders? & Polyphytophagous. \\
\hline 36 & - & $? ?$ & Foliage feeders? & $\begin{array}{l}\text { Need to be studied. May be important due to } \\
\text { its specificity. }\end{array}$ \\
\hline 37 & - & $? ?$ & Foliage feeders? & $\begin{array}{l}\text { Need to be studied. May be important as } \\
\text { restricted to some Asclepiadoideae. }\end{array}$ \\
\hline 38 & - & $? ?$ & Foliage feeders & $\begin{array}{l}\text { Need to be studied. There are no records of } \\
\text { species of this family in the genera studied. }\end{array}$ \\
\hline 39 & - & occasional? & Sap feeders & $\begin{array}{l}\text { Need to be studied. There are no records of } \\
\text { species of this family in the genera studied. }\end{array}$ \\
\hline 17 & 43 & common & Foliage feeders & Polyphytophagous. \\
\hline 40 & 15 & rare & Flower feeders? & $\begin{array}{l}\text { Need to be studied. There are no records of } \\
\text { species of this family in the genera studied. }\end{array}$ \\
\hline
\end{tabular}

Table 3. Comments on parasite and predator populations found on different species of Araujia. Note: Frequency: see previous table. (23): Collecting site.

\begin{tabular}{|c|c|c|c|}
\hline Taxon & Common name & Frequency & Comments \\
\hline Lebia securigera. & Ground beetles & Rare (23) & On Araujia hortorum \\
\hline Adalia bipunctata & Ladybirds & Rare (DLC) & On Araujia odorata \\
\hline Azya luteipes & Ladybirds & Occasional (GON) & $\begin{array}{l}\text { On Araujia hortorum } \\
\text { Predating Aphis nerii on }\end{array}$ \\
\hline Coccinella ancoralis & Ladybirds & $\begin{array}{c}\text { Common }(6,8,22,23,193 \text {, } \\
\text { 196, FRIG. SUR) }\end{array}$ & $\begin{array}{l}\text { Araujia odorata, A. horto- } \\
\text { rum, A. angustifolia and } \\
\text { Funastrum gracile }\end{array}$ \\
\hline Harmonía axyridis & Ladybirds & Occasional (21) & On Araujia hortorum \\
\hline Cycloneda sanguinea & Ladybirds & Common (20) & On Araujia angustifolia \\
\hline Staphilinidae & Rove beetles & Rare (IV) & On Araujia hortorum \\
\hline Podisus aenescens & Spined soldier bug & Rare $(208,8 M)$ & $\begin{array}{c}\text { On Philibertia latiflora } \\
\text { and Araujia sericifera }\end{array}$ \\
\hline Zelus leucogrammus & Assassin bug & Rare (197) & On Philibertia latiflora \\
\hline Mutillidae & Ant Wasps & rare & On Araujia odorata \\
\hline Serie Parasítica & Parasitic wasps & rare? & On Araujia hortorum \\
\hline Pompilidae & Wasps & rare & On Araujia odorata \\
\hline
\end{tabular}

\section{BIBLIOGRAPHY}

Bosq, J.M. 1937. Lista preliminar de los hemípteros (heterópteros), especialmente relacionados con la agricultura nacional. Revista de la Sociedad Entomológica Argentina 9: 111-134.

Bosq, J.M. 1940. Lista preliminar de los hemípteros (heteropteros), especialmente relacionados con la agricultura nacional. (Continuación). Revista de la Sociedad Entomológica Argentina 10: 399-417.

Bosq, J.M. 1943. Segunda lista de coleópteros de la República Argentina dañinos a la agricultura. (Reedición de Ingeniería Agronómica, vol IV, 1822, Buenos Aires 1942). Revista del Ministerio de Agricultura Nacional, Dirección de Sanidad Vegetal 4: 1-80.

Cordo, H.A., G. Logarzo, K. Braun \& O.R. Di Iorio. 2004. Catálogo de Insectos Fitófagos de la Argentina y sus Plantas Asociadas. South American Biological Control Laboratory USDA-ARS - Sociedad Entomológica Argentina. 720 pp.

Meyer, T. 1944. Asclepiadaceae. En: H.R. Descole (Ed.), Genera et Species Plantarum Argentinarum 2. Kraft, Buenos Aires.

Rapini, A., J. Fontella Pereira \& D.J. Goyder. 2011. Towards a stable generic circumscription in Oxypetalinae (Apocynaceae). Phytotaxa 26: 9-16.

Villamil, C., R. Dehley \& S. Villamil. 2005. Preliminary studies on the Araujia hortorum-sericifera complex in Argentina. Landcare Research Report 2005.

Villamil, C., R. Dehley \& D.L. Carpintero. 2006. Preliminary studies on the Araujia hortorum-sericifera complex in Argentina. Landcare Research Report 2005-2006.

Winks, C.J. \& S.V. Fowler. 2000. Prospects for Biological Control of Moth Plant, Araujia sericif- 
era (Asclepiadaceae). Landcare Research Contract Report: LC 9900/100. 18 pp.

Winks, C.J., N.W. Waipara \& A.F. Gianotti. 2004.
Invertebrates and Fungi Associated with Moth Plant, Araujia sericifera, in New Zealand. Landcare Research Contract Report LC 0405/009. 29 pp.

Recibido: 19-X-2012

Aceptado: 12-XI-2013 